\title{
Evaluation of a New Balloon Catheter for Difficult Calcified Lesions in Infrainguinal Arterial Disease: Outcome of a Multicenter Registry
}

\author{
G. J. Spaargaren - M. J. Lee · J. A. Reekers $\cdot$ H. van Overhagen · \\ L. J. Schultze Kool · Y. L. Hoogeveen
}

Received: 1 February 2008/ Accepted: 25 June 2008/Published online: 26 July 2008

(C) The Author(s) 2008

\begin{abstract}
The purpose of this study was to assess the technical performance and immediate procedure outcome of a new balloon catheter in the treatment of calcified lesions in infrainguinal arterial disease. Seventy-five patients with infrainguinal arterial disease were prospectively entered into the registry. The catheter (ReeKross Clearstream, Ireland) is a 5- to 6-Fr balloon catheter with a rigid shaft intended for enhanced pushability. Only technical procedural outcome was recorded. Treated calcified lesions (range: 5-30 cm), assessed angiographically, were located in the superficial femoral, popliteal, and crural arteries. In 67 patients the lesion was an occlusion. Guidewire passage occurred subintimally in 68 patients. In 24 patients a standard balloon catheter was chosen as first treatment catheter: 5 failed to cross the lesion, 8 balloons
\end{abstract}

G. J. Spaargaren · L. J. Schultze Kool · Y. L. Hoogeveen Department of Radiology, Radboud University Nijmegen Medical Centre, Geert Grooteplein 10, 6525 GA Nijmegen, The Netherlands

M. J. Lee

Department of Radiology, Beaumont Hospital, Beaumont Rd., Dublin 9, Ireland

\section{J. A. Reekers}

Department of Radiology, Amsterdam Medical Centre, Amsterdam, Meibergdreef 9, 1105 AZ Amsterdam, The Netherlands

H. van Overhagen

Department of Radiology, Leyenburg Hospital, Leyweg 275,

2545 CH Den Haag, The Netherlands

\section{Y. L. Hoogeveen $(\bowtie)$}

Department of Radiology, UMC St Radboud, 667, Box 9101, 6500 HB Nijmegen, The Netherlands

e-mail: y.hoogeveen@rad.umcn.nl ruptured, and in 11 patients there was an inadequate dilatation result. In only one of the five patients did subsequent use of the ReeKross catheter also fail in lesion crossing. The ReeKross was successful as secondary catheter in the other 23 cases. In 50 patients the ReeKross was used as primary catheter. In total the ReeKross crossed the lesions in 74 patients. After passage and dilatation with this catheter in 73 patients ( 1 failed true-lumen reentry), 19 had $>30 \%$ residual lesions, of which 11 were not treated and 8 were successfully stented. No ReeKross balloons ruptured. We conclude that in the treatment of difficult calcified lesions in arterial stenotic or occlusive disease, the choice of a high-pushability angioplasty catheter, with more calcification-resistant balloon characteristics, like the ReeKross, warrants consideration.

Keywords Percutaneous transluminal angiopasty · Infrainguinal · Occlusion · Registry $\cdot$ Recanalization

\section{Introduction}

Although there is now a strong tendency to treat more severe and extensive arterial occlusive disease below the inguinal ligament percutaneously instead of surgically [1, 2], heavy intima and media calcification may inhibit balloon catheter passage distally after guidewire passage, resulting in treatment failure. In cases where intraluminal recanalization is not possible, the now established technique of subintimal angioplasty is used in the treatment of long vascular occlusions [3]. The latter involves crossing the lesion using a guidewire loop technique and balloon catheter. However, in calcified lesions the advance of devices may fail. Heavy calcifications may also cause balloon puncture. Percutaneous treatment success appears 
to be limited not only by the characteristics of the involved lesions but also by the characteristics of the traditional balloon catheters. The failed recanalization attempt in approximately $20 \%$ of cases [4] indicates its inability to overcome these intrinsic vessel limitations.

We assessed the technical performance and immediate procedure outcome of a new balloon catheter intended for angioplasty of heavily calcified arterial disease in a multicenter registry in patients with occlusive arterial disease below the inguinal ligament.

\section{Materials and Methods}

\section{Patients}

Seventy-five patients presenting with infrainguinal stenotic or occlusive disease (classified as Fontaine III) scheduled for routine percutaneous treatment and in whom the Reekross catheter (ClearStream Technologies Ltd., Wexford, Ireland) was used as either primary or secondary angioplasty catheter were entered into a multicenter registry at four European centers from December 2006 to June 2007. The multicenter registry was prospective and nonrandomized.

The choice of the initial angioplasty catheter to be used for the procedure was determined by the interventional radiologist. There were no special exclusion criteria associated with the registry, only those related to contrast or pregnancy. No patient demographic data were recorded, only anatomical, procedural, and technical data. The registry did not require IRB approval. There were no commercial interests involved in performing this study.

\section{Device Description}

The ReeKross balloon catheter is a commercially (outside the USA) available 5- to 6-Fr over-the-wire system that consists of a rigid shaft, which extends from the proximal hub to the distal tip of the catheter, and an extra rugged inflation lumen. The rigidity of the shaft is achieved by a hypotubing technology. The balloon is a blend of nylon/ pebax, developed using the so-called QuadFlex technology, which the manufacturer asserts affords the balloon with the necessary characteristics to perform multiple inflations while advancing the catheter through long occluded lesions. Radiopaque bands indicate the location of the balloon under fluoroscopy. Balloon lengths range from 4 to $22 \mathrm{~cm}$, with diameters of $2.0-6.0 \mathrm{~mm}$. Rate burst pressure is given as 14-18 atm for 4-cm-long balloons and 13$15 \mathrm{~atm}$ for 8 - to $22-\mathrm{cm}$-long balloons. The catheter is available in shaft lengths of $75,110,130$, and $140 \mathrm{~cm}$ and is compatible with a guidewire of maximum 0.035 -in. diameter. According to the manufacturer, the balloon catheter is suitable for both intraluminal and subintimal angioplasty.

\section{Procedure and Technical Data Collection}

Data were collected on the following: location and length of stenosis/occlusion, presence of calcification, subintimal/ intraluminal passage, primary or assisted use of the ReeKross catheter for dilatation, number of balloon inflations, balloon puncture or rupture, postdilatation procedure results, and any additional treatment performed. Dilatation pressures were not recorded. Any technical anomalies and adverse events occurring during the procedure were recorded.

\section{Results}

In 67 of the 75 patients there was total occlusion. Calcified lesions were located in the superficial femoral artery (SFA; $n=36)$, popliteal artery $(n=23)$, and crural arteries $(n=48)$. Lesions were located in two or more vessels in 29 patients. Median lesion lengths were $15 \mathrm{~cm}$ (range: 5$25 \mathrm{~cm})$ for SFA, $10 \mathrm{~cm}(5-12 \mathrm{~cm})$ for popliteal artery, and $11.5 \mathrm{~cm}(5-30 \mathrm{~cm})$ for crural vessels (Table 1).

An antegrade approach was used in all procedure, as was the use of a Terumo 0.035-in. guidewire. Guidewire passage was subintimal in 68 patients and intraluminal in 6 , and no guidewire passage was possible in 1 patient, either intraluminal nor subintimal.

In 24 of 74 patients other balloon catheters were used prior to the ReeKross catheter. In one patient, both the original balloons ruptured. This resulted in 8 events of rupture of the original balloon (all OPTA; Cordis, a J\&J Company) - of which 5 were in the SFA (average lesion

Table 1 Treated lesion locations and lengths

\begin{tabular}{lllllll}
\hline Length & $\begin{array}{l}\text { SFA } \\
(n=36)\end{array}$ & $\begin{array}{l}\text { Popliteal } \\
(n=23)\end{array}$ & $\begin{array}{l}\text { Truncal } \\
(n=9)\end{array}$ & $\begin{array}{l}\text { ATA } \\
(n=23)\end{array}$ & $\begin{array}{l}\text { ATP } \\
(n=4)\end{array}$ \\
\hline Mean $(\mathrm{cm})$ & 14.9 & 9.7 & 3.7 & 16.8 & 11.8 & $\begin{array}{l}\text { Peroneal } \\
(n=12)\end{array}$ \\
Median (cm) & 15.0 & 10.0 & 4.0 & 17.0 & 11.0 \\
Range (cm) & $5-25$ & $5-12$ & $3-4$ & $10-30$ & $10-15$ \\
\hline
\end{tabular}

Note: SFA, superficial femoral artery; ATA, anterior tibial artery; ATP, posterior tibial artery 
length, $11.2 \mathrm{~cm}), 1$ popliteal $(12 \mathrm{~cm})$, and 2 infrapopliteal $(11.0 \mathrm{~cm})-5$ failures in crossing the lesion, and 12 cases wherein the original catheter gave an inadequate postdilatation result. In only one of the five patients did subsequent use of the ReeKross catheter also fail in lesion crossing. The ReeKross was successful in completing the procedure as secondary catheter in the other 23 cases.

The ReeKross was used as the primary, and only, treatment catheter in 50 patients. In one case as primary catheter, subintimal passage across the lesion was successful but true-lumen entry was not achieved, thus dilatation was not performed. Therefore, in total, this catheter crossed the lesion in 74 of 74 patients where guidewire passage was achieved, with 73 dilatations performed. Postdilatation results in 54 of these 73 patients showed $<30 \%$ residual stenosis. In the 19 patients with $>30 \%$ residual stenoses, 8 lesions were treated by stent placement, while the remaining 11 were not treated. With the intention to treat patients with the ReeKross catheter as primary catheter, the technical success (no adjunctive treatment/stent) rate was $82 \%$ (42 of 51 patients; 1 patient failed reentry, and 8 patients required a stent). Overall, the assisted angiographic success after adjunctive stent placement was $97 \%$ ( 73 of 75 patients). The average number of inflations per treated patient with the ReeKross catheter was 2.9 (range: 1-8 inflations). There were no
ReeKross balloon ruptures or punctures in any of the treated patients. There were no device-related clinical adverse events reported.

\section{Discussion}

This registry indicates that the ReeKross catheter is an applicable instrument in potentially difficult procedures in the treatment of occluded or stenotic arterial disease, with a low risk of technical failures and an acceptable angiographic and immediate procedure outcome in the majority of patients.

All procedures in this registry were by antegrade approach. The stiffness of the catheter shaft makes it unsuitable for a retrograde procedure over the bifurcation.

Although this registry study allows limited direct comparison to other balloon catheters, in cases where technical failures occurred with a standard balloon catheter, it does indicate the potential applicability of a catheter with high pushability and equipped with a more durable balloon. These characteristics may also have a positive effect on limiting the procedure time in potentially difficult recanalizations.

In patients who present with severe occlusive peripheral disease, recanalization almost always takes the subintimal
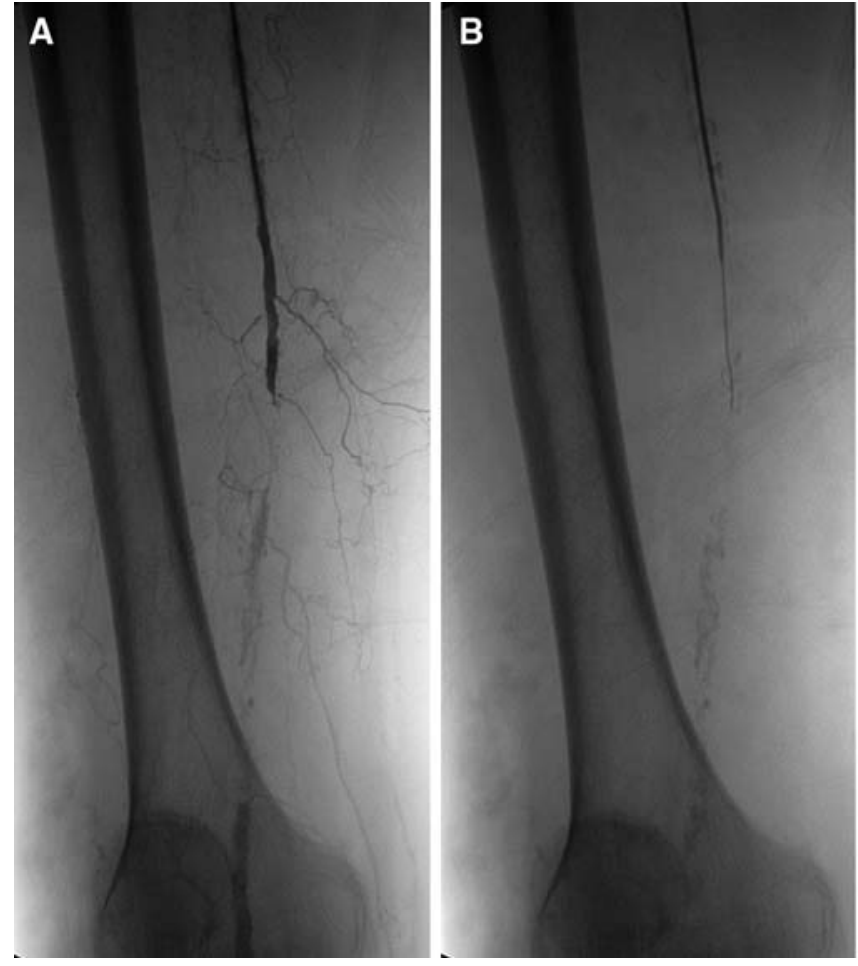

Fig. 1 (A) Patient with total occlusion of the right distal superficial femoral artery (SFA)-popliteal artery with severe calcifications, collaterals, and a patent popliteal artery; (B) subintimal passage of
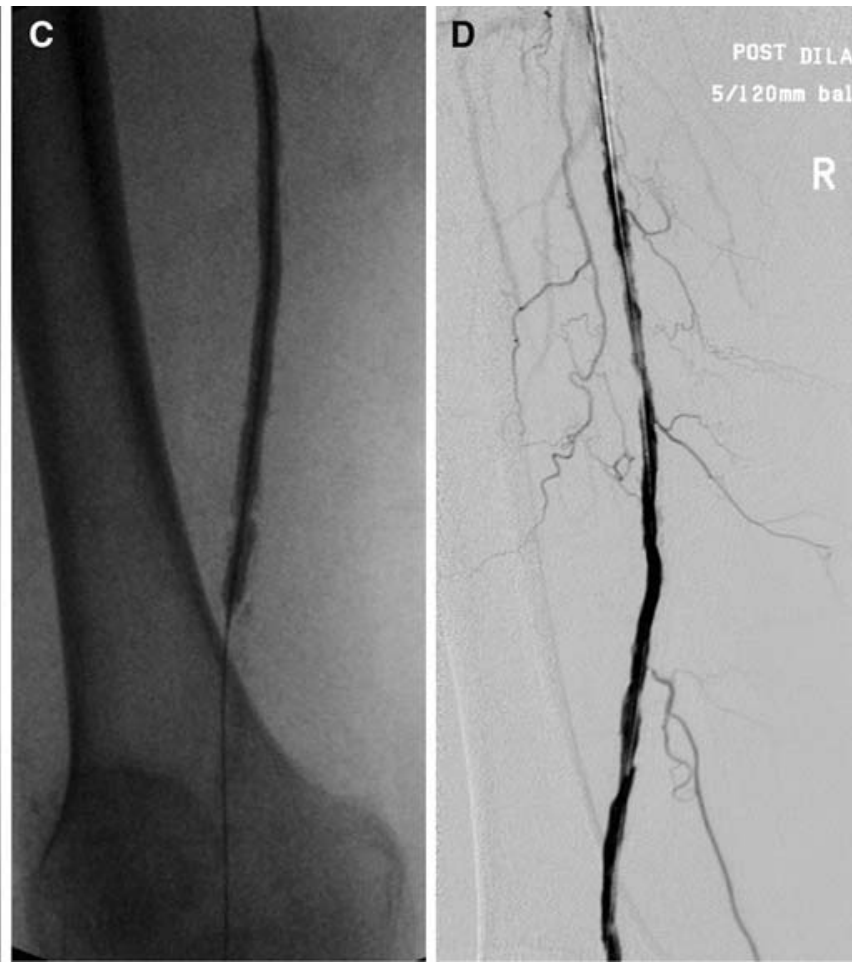

the guidewire; $(\mathbf{C})$ subsequent dilatation with a $5 \times 120$-mm balloon; and (D) final angiographic result 
route, as intraluminal access is not available. This technique has allowed the treatment of lesions otherwise not crossable using standard transluminal approaches $[3,5,6]$. In this registry, the majority of recanalizations were subintimal (Fig. 1). The ReeKross catheter showed a high immediate angiographic and procedural success rate in all these patients, without catheter-related clinical adverse events, thereby indicating its suitability for this procedure.

The outcome of this registry indicates that this catheter can be a valuable addition to the arsenal of devices for percutaneous treatment of difficult heavily calcified infrainguinal disease, possibly expanding the range of treatable lesions. Verification of the latter requires a prospective double-blind study with conventional balloon catheters.

Open Access This article is distributed under the terms of the Creative Commons Attribution Noncommercial License which permits any noncommercial use, distribution, and reproduction in any medium, provided the original author(s) and source are credited.

\section{References}

1. Nasr MK, McCarthy RJ, Hardman J et al (2002) The increasing role of percutaneous transluminal angioplasty in the primary management of critical limb ischemia. Eur J Vasc Endovasc Surg 23:398-403

2. Adam DJ, Beard JD, Cleveland T et al (2005) BASIL trial participants bypass versus angioplasty in severe ischaemia of the leg (BASIL): multicentre, randomised controlled trial. Lancet 3:1925-1934

3. Markose G, Bolia A (2006) Subintimal angioplasty in the management of lower limb ischaemia. J Cardiovasc Surg (Torino) 47:399-406

4. Rogers JH, Laird JR (2007) Overview of new technologies for lower extremity revascularization. Circulation 116:2072-2085

5. Kim JS, Kang TS, Ahn CM et al (2006) Efficacy of subintimal angioplasty/stent implantation for long, multisegmental lower limb occlusive lesions in patients unsuitable for surgery. J Endovasc Ther 13:514-521

6. Landis GS, Faries PL (2007) New techniques and developments to treat long infrainguinal arterial occlusions: use of reentry devices, subintimal angioplasty, and endografts. Perspect Vasc Surg Endovasc Ther 19:285-290 